\title{
Rebecca Anouche singt Aznavour
}

\section{Gerhild FUCHS (Innsbruck)}

Nach dem großen Erfolg des ersten, im Mai 2018 in Innsbruck abgehaltenen Chansonabends mit Rebecca Anouche und ihrer der „French World Music“ verschriebenen Formation ${ }^{1}$ organisierte das Institut Franco-Tyrolien d'Innsbruck (IFTI) unter der Leitung von Béatrice Gaigg für den 8. Oktober einen neuerlichen Auftritt der Sängerin, welcher diesmal ganz im Zeichen der französischen Chanson-Legende Charles Aznavour stehen sollte. Dass dieses Konzert zugleich als Nachruf auf den großen Meister fungieren würde, der sieben Tage zuvor - am 1. Oktober - verstorben war, konnte man zum Zeitpunkt der Organisation freilich noch nicht wissen.

Im glänzend besuchten Saal der Bäckerei-Kulturbackstube (wohl die schönste der ,alternativen' Konzert-Locations in Innsbruck ${ }^{2}$ ) präsentierte sich die in Graz ansässige Rebecca Anouche diesmal mit nur zwei Begleitmusikern: dem Akkordeonisten und Pianisten Manu Mazé, der auch für die Arrangements verantwortlich zeichnete (und der auch fixes Mitglied von Anouches anderen Formationen ist), sowie der ansonsten bei den Grazer Philharmonikern tätigen Cellistin Ivanila Lultcheva.

Als gebürtige Pariserin mit armenischen Wurzeln, deren Familie (wie sie im anschließenden Interview erzählte) mit jener Aznavours in Paris auch bekannt war, besitzt Anouche quasi von vornherein eine gewisse Nähe zu dem großen Chansonnier. Es ist zum Teil wohl dieser Affinität, vor allem aber der generellen Sensibilität und Wandlungsfähigkeit der Sängerin sowie der hochstehenden Qualität ihrer musikalischen Begleiter zu verdanken, dass der Konzertabend zu einer in jeder Hinsicht würdigen Hommage an Charles Aznavour geriet. Aus der Liste der Chansons, die aus dessen großem Repertoire von Anouche und Mazé für den Abend ausgewählt worden waren, ${ }^{3}$ sollen hier nur einige Highlights kurz angesprochen werden.

Als solche können insbesondere jene Lieder gelten, die sich entweder durch die Besonderheit der Arrangements oder durch die theatralischen Inszenierungen und das schauspielerische Talent der Interpretin ins Gedächtnis eingruben. Zuerst zu letzterem: Tatsächlich versteht es Anouche, ihr Outfit mit Hilfe diverser Accessoires vor den Augen des Publikums teilweise in Sekundenschnelle zu verändern. $\mathrm{Zu}$ einem auch komödiantischen Höhepunkt des Konzerts geriet auf diese Weise „Tu te laisses aller“, wo die Interpretin sich gewissermaßen doppelte, indem sie gesanglich die Rolle des vorwurfsvollen Gatten und mit ihrem Outfit (Lockenwickler, Morgenrock, etc.) zugleich jene der sich gehenlassenden Ehefrau mimte. Was somit bei diesem und bei etlichen anderen Chansons deutlich vor Augen trat, 
war die von Anouche in den kurzen Einleitungen zu den einzelnen Liednummern betonte Qualität vieler von Aznavours Songs als ,Miniaturfilme', die auf prägnante und immer auch poetische Weise eine Geschichte erzählen. Als gelungene musikalische Miniaturfilme konnte die Sängerin des Weiteren auch „La mamma“ präsentieren, das mit seinem liebevollsatirischen Blick auf die Besonderheiten einer migrantischen Community besticht, oder den Hit „Mes amis, mes amours, mes emmerdes“ mit seiner für Aznavour so typischen Mischung von Ausgelassenheit und Melancholie, sowie schließlich das großartige „Comme ils disent", mit dem die Sängerin eine eindrucksvolle, gleichermaßen mit Feingefühl und Inbrunst vorgetragene Kostprobe aus Aznavours ernsthaftem, von einer Art zärtlicher Tragik geprägten Repertoire gab. Sie betonte in der Einleitung zu dieser Nummer das mutige Engagement des Künstlers, stammt dieses in der Ich-Form verfasste Porträt eines traurigen Travestie-Künstlers doch aus einer Zeit (1972), in der Homosexualität in Frankreich noch strafbar war.

Neben ihrer armenischen Abstammung und ihrem schauspielerischen Talent weist die Sängerin Anouche mit dem großen Chansonnier noch eine weitere Gemeinsamkeit auf, nämlich ihre Mehrsprachigkeit. Diese erlaubte es ihr, einige der Chansons wie "La bohème“ oder „Hier encore“ („Was auch kommt im Leben“) in deutschen Versionen vorzutragen auf denen schließlich auch Aznavours enormer Erfolg im deutschsprachigen Raum beruhte. In einwandfreiem Englisch gab die Sängerin den Welthit „She“ zum Besten, und in einem gelungenen Gemisch aus Deutsch, Französisch und Englisch gab es das nicht weniger berühmte „Formidable“ zu hören.

Die zuletzt genannte Nummer kann auch als herausragendes Beispiel für die ganz generell ausgezeichneten musikalischen Arrangements dieses Aznavour-Abends angeführt werden. So ließ Manu Mazé am Beginn des von ihm drängend-rhythmisch umgesetzten Songs das allseits bekannte Motiv aus Khatschaturjans Säbeltanz erklingen, was abgesehen von der musikalischen Passgenauigkeit auch aus der armenischen Herkunft beider Künstler, Aznavours und Khatschaturjans (sowie nicht zuletzt Rebecca Anouches), seine Motivation bezog. Die Klangwelt der Levante war auch in „Les deux guitares“(„Spiel Zigeuner“) perfekt eingefangen, während überraschend eingesetzte Swing-Einsprengsel die Melancholie von "Hier encore" auf gelungene Weise konterkarierten.

Eine Qualität des Konzerts, die abschließend noch hervorgehoben werden soll, bestand gerade darin, dass die Virtuosität der Interpretin, der beiden Musiker oder des Arrangeurs sich nie exklusiv in den Vordergrund stellten, sondern vielmehr der Charakter einer achtungsvollen Hommage durchgehend gewahrt wurde. Mit den ausgewählten Liedinterpretationen gelang es dem Ensemble, Charles Aznavour als jenen Künstler erscheinen zu lassen, als den ihn Rebecca Anouche in ihren Kommentaren während des Konzerts sowie im anschließenden Interview nachdrücklich präsentierte: Als großen Poeten unter den klassischen Chansonniers und als Autor von ,musikalischen Miniaturfilmen', die neben melancholischen und humoristischen Tönen fallweise durchaus auch Tabu-Themen anklingen lassen. 


\section{Endnoten}

1 http://www.rebecca.co.at/french-world-music.php (Zugriff 12.11.2018).

2 http://www.diebaeckerei.at/ (Zugriff 12.11.2018).

3 „Je me voyais déjà “, „Emmenez-moi“, „She“, „La bohème“, „Hier encore“, „Comme ils disent“, „Tu te laisses aller“, „Formidable“, „La mamma“, „Mes emmerdes“, „Il faut savoir“, „Les deux guitares“, „Le temps“. 